\title{
FE Analysis of Contact between Residual Limb and Socket during Simulation of Amputee Motion
}

\author{
Giorgio Colombo ${ }^{1}$, Roberto Morotti ${ }^{2}$ and Caterina Rizzi ${ }^{3}$ \\ ${ }^{1}$ Polytechnic of Milan, giorgio.colombo@polimi.it \\ ${ }^{2}$ University of Bergamo, roberto.morotti@unibg.it \\ ${ }^{3}$ University of Bergamo, caterina.rizzi@unibg.it
}

\section{INTRODUCTION}

In modular prosthesis, the socket is the custom-fit part manufactured around the patient's residual limb. It is the most critical component from which depends the whole prosthesis functionality; therefore the key issue is to define its shape in order to ensure the best comfort and fit.

Nowadays, the prosthesis design and manufacturing are still carried out manually and deeply relies on the prosthetic's skill and experience. This subjective and static assessment causes a high rate of inappropriate prosthetic devices, increasing costs and times.

In such a context, modern imaging techniques and computer-aided tools can help to gain higher quality in the prosthetic socket and allow a more objective design and manufacturing process. On the market we can find some prosthetic CAD/CAM sys-tems $[1,3,17,18]$ that allow creating the $3 \mathrm{D}$ socket model onto the digital external shape of the resid-ual limb (acquired by Reverse engineering technique) and using standard libraries. However, they partially digitalize the socket design process, do not provide any support or guidance to the designer and are not integrated with simulation tools, such as FEA (Finite Element Analysis) or multi-body systems, to evaluate the design quality.
To overcome these limits, we developed a vir-tual environment, called Prosthesis Modeling Lab, where the orthopedic technician can design the whole prosthesis both for transtibial and transfemoral amputees. S/he directly creates the 3D socket model onto the residual limb digital model, which includes bony structure and soft tissues, using a dedicated CAD tool, named Socket Modeling Assistant-SMA [7]. The system guides the prosthetist during each design task providing specific knowledge and rules (e.g., selection rules for standard parts or where and how to modify the socket shape), coherently with the traditional procedures.

In [5] we verified the possibility to implement an automatic procedure for Finite Element Analysis focusing the attention on transtibial amputee in static load condition with the aim of verifying contact pressure and then optimising the socket shape. In this work we took two steps forward: 1) the extension of FE model to transfemoral case, and 2) the integration of FEA with Human Modeling in order to analyze the pressure distribution over residual limb surface in a posture or during different gait conditions. In fact, to achieve better design solutions, it is necessary to face the variable contact conditions between the residual limb and socket during amputee's motion. To this end, we have combined two main phases: first the 
gait analysis using the patient's avatar of an unilateral transfemoral male amputee, then the FE contact analysis between the residual limb and socket considering loads distribution computed in the first phase. Therefore, we put the attention on the integration of virtual gait analysis with the FE analysis.

In this paper, we first explain in detail our approach and, then, the two main steps, namely the virtual gait simulation and FE contact pressure analysis. Finally, preliminary experimentation performed with a transfemoral case study and results reached so far are reported.

\section{THE APPROACH}

The design platform, shown in Fig. 1., is centered on the 3D digital model of the amputee and it works both for transtibial and transfemoral prosthesis. It integrates ad hoc tools to acquire patient's data, human modeling system to perform gait analysis, CAD tools to model prosthesis components, both standard and custom-fit, and FEA package to analyze the socketresidual limb interaction.

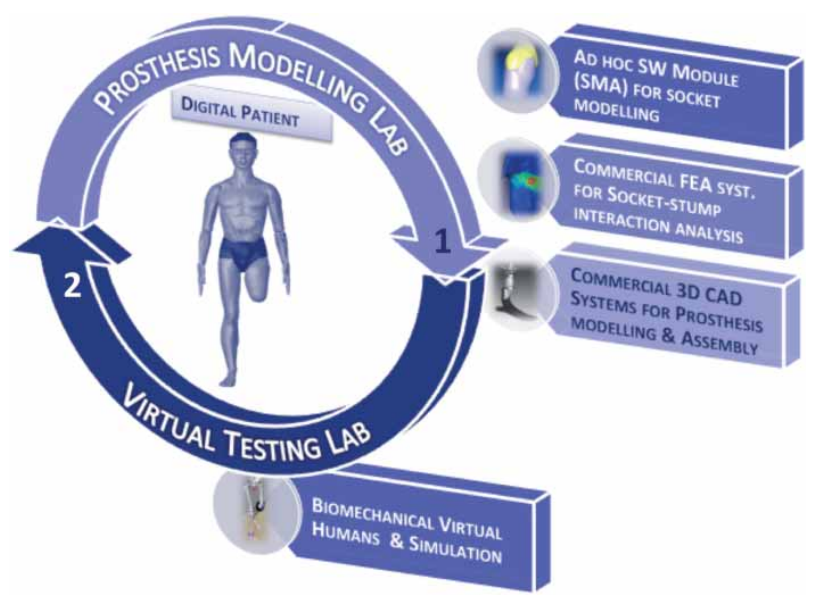

Fig. 1: Prosthesis design platform.

The challenge is to design, manufacture and assembly an optimal prosthesis with a well fitting socket, which distributes loads over desired regions of the residual limb. Commonly, socket evaluation is made by analyzing the interface pressure in critical regions: pressure values should not exceed the respective pain threshold in order to be tolerated for a certain time [22]. This means that the meaningful parameter recognized to evaluate the socket shape is the contact pressure between socket and residual limb during patient's walking, since it allows taking into account the whole residual limb's morphology.
Fig. 2. portrays the logic scheme of proposed approach to validate socket design where:

- First patient's history (i.e., a set of characteristics usually considered by the prosthetist) and residual limb geometry are acquired. The former is necessary for both prosthesis modeling and avatar characterization. The latter is acquired using MRI technology and its 3D model is reconstructed using an ad hoc module that permits to automatically generate the 3D model of the residual limb (soft tissues and bones).

- The prosthetist models the socket directly onto the external surface of the residual limb applying in automatic or semi-automatic way modeling procedures and design rules suggested by SMA. The socket-residual limb alignment is guaranteed using the same coordinate system adopted inside the SMA, and kept also in the following activities.

- Once modeled and assembled the complete prosthesis, the patient's avatar is created according to patient's anthropometric measures and the gait analysis is performed to get the forces acting on the socket. The LifeMOD human modeling system is used to generate and simulate the patient's avatar.

- SMA and LifeMOD release all data necessary to characterize the FE model and run the script (written in Python language) to create the FE model without any human intervention.

- FE solver (in our case Abaqus) automatically executes the analysis and generates the output file containing the pressure values.

- Analysis results are imported into SMA and visualized with a color map. SMA evaluates pressure distribution and highlights the zones that should be modified.

Through an iterative process of adjustments, the socket shape can be modified and optimized by prosthetist to distribute loads in the appropriate way and minimizing weight. Once the pressures map on the residual limb is consistent with the adopted parameters, the socket shape can be manufactured.

\section{VIRTUAL HUMAN MODEL}

During last years, the product development process is often supported by human modeling tools, and their application is increasingly important, especially for product ergonomics studies $[6,15]$. It's possible to identify four main categories of tools according to the type of application: virtual human/actors for entertainment, mannequins for clothing, virtual humans for ergonomic analyses, and biomechanical models. Our focus is on the last category, the best suited for 


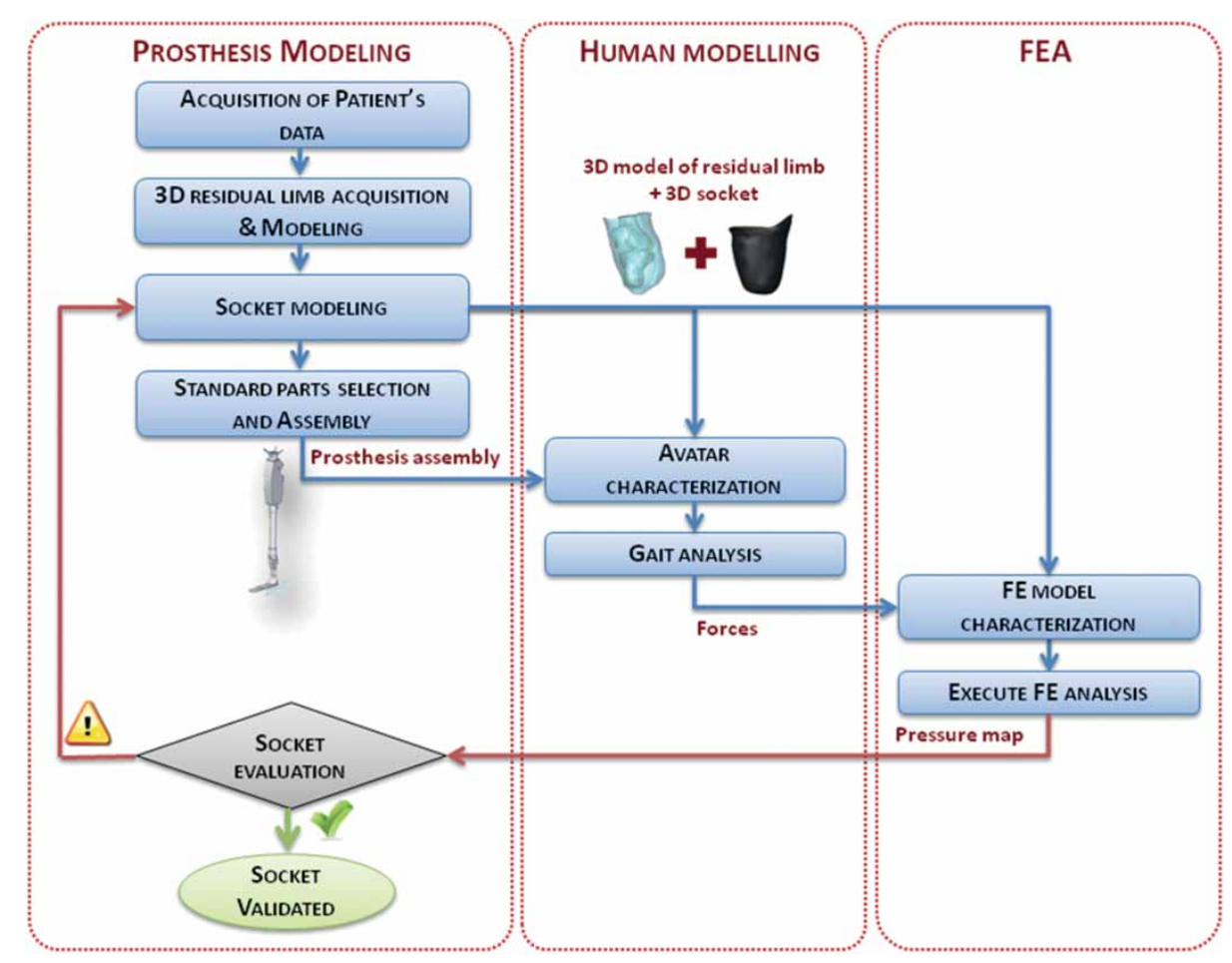

Fig. 2: Logic schema of the approach.

prosthetic field, because it permits to simulate accurately the human body movement, also considering different pathological conditions or disabilities.

In the following sections, we describe the steps necessary to get the forces acting on the socket during walking, from the implementation of patient's avatar to gait simulation.

\subsection{Patient's Avatar}

The patient's avatar is a customized instance of LifeMOD, a biomechanical modeling package based on MSC ADAMS system. It permits to create a detailed biomechanical model of a human body using rigid links connected through joints to simulate the skeleton and flexible elements to represent muscles, tendons and ligaments.

The patient's avatar wearing the prosthesis is created in two steps. Starting from the LifeMOD standard model of the human being, we first generate a customized virtual model of the amputee according to his/her anthropometric measures and replacing the leg with 3D model of the residual limb (femur and soft tissues), as shown in Fig. 3(a). The femoral head is linked to the avatar's hip and then soft tissues are properly placed. In the second step the 3D prosthesis is imported using Parasolid format and the correct positioning of each part is obtained taking into account the prosthesis height and foot rotation respect to the vertical line. In particular, the socket alignment respect to soft tissues is guaranteed using the same coordinate system adopted inside the SMA; while, the prosthetic foot is aligned symmetrically to the other one (Fig. 3(b).).

\subsection{Walking Simulation}

Patient's walking has been simulated using motion laws deduced from experimental data acquired with a marker less Motion Capture (Mocap) equipment, based on four Sony-eye webcams [4]. The motion law is described by "Motion agents" that drive the skeleton joints and teach to patient's avatar how to move. Once position and orientation of each avatar's links are known and traced, LifeMOD proceeds with the inverse dynamics simulation to get internal forces, torques and ground reactions.

In our case, to drive the motions of the artificial leg, we associated three "Motion Agents" to the prosthetic foot, one to the prosthetic knee and another one to the femoral head. Furthermore, in order to complete the kinematic chain, we inserted a rigid link aligned with the femur between the hip and the knee joints, modelling the socket and residual limb fitted together.

Main goal of the simulation is to evaluate forces transmitted on the stump-socket-prosthesis system during walking, and in particular, loads acting on the socket. We focused the attention on the first step of the patient's walking, when s/he starts to walk, because it's the most challenging in terms of involved forces. We consider the patient's avatar during loading step in three different stance phases: initial loading response, midstance, and terminal 

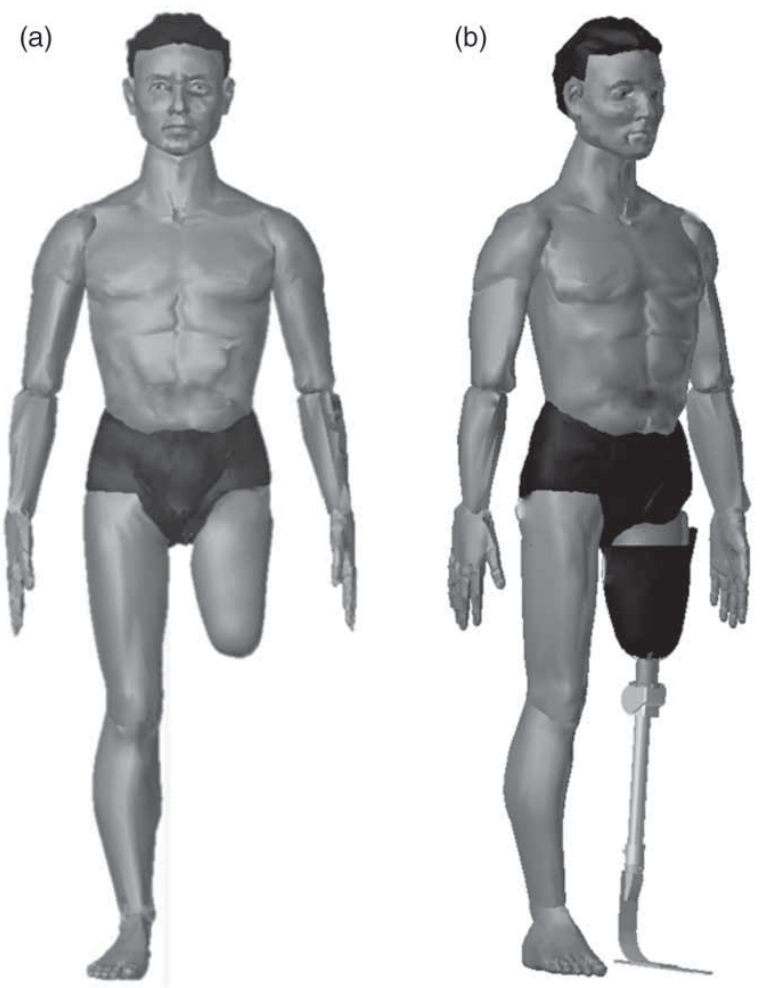

Fig. 3: (a) Patient's avatar; (b) avatar wearing the prosthesis.

stance as shown in Fig. 4. Specifically, to avoid convergence problems in FE analysis, we neglect the first and last frames of the stance phase where the load values are lower than $25 \%$ of the magnitude peak. Fig. 5. shows the filtered numerical results of the three force components, each exported separately in a text file, and the magnitude over the first stance phase.

\section{FE MODEL}

Finite element modeling and analysis to evaluate the socket-residual limb interaction has been performed since the 1990s [2,16,20,25,28]; most of researches considered transtibial socket, only few transfemoral. Anyway, none of them tried to integrate and/or automate the analysis within a CAD framework and mainly focused on the fulfillment of the model itself. Instead, our main goal has been to implement an automatic simulation procedure to make the prosthetist able to run automatically FE analysis and validate socket design and functionality.

Among the various FE solvers commonly used in this field, we adopted Abaqus package V 6.9 (Dassault Systemes S.A.) that permits to bypass the graphical user interface Abaqus/CAE and communicate directly with the Abaqus Kernel through a Python script. The Abaqus kernel interprets the script commands and automatically creates an internal representation of the model.

To achieve our purpose, we first identified a running model using the Abaqus/CAE GUI. Abaqus converts in a script command every graphical operation, option or settings and stores them in a file (.jnl). From this, we generalized the commands to obtain a script that automatically implements the FE model and executes the analysis applying the loads computed during the avatar's gait simulation.

In the following, solutions adopted to realize a FE model for the transfemoral case are summarized.

The socket is imported in Abaqus as 3D shell, because socket thickness is considerably smaller than the other two dimensions, while bony structure and soft tissues as 3D solids. These parts are aligned within the SMA before starting the socket design. Bones and soft tissues are merged to form a single part, the residual limb, without geometric discontinuity but characterized by two different models material.

Because of the automatic procedure a free auto meshing technique has been adopted. We used 3noded triangular (S3R) elements for the socket and 4-node tetrahedral (C3D4) for the residual limb, which increase their size in the internal regions. The seed values, summarized in Tab. 1., has been chosen (a)

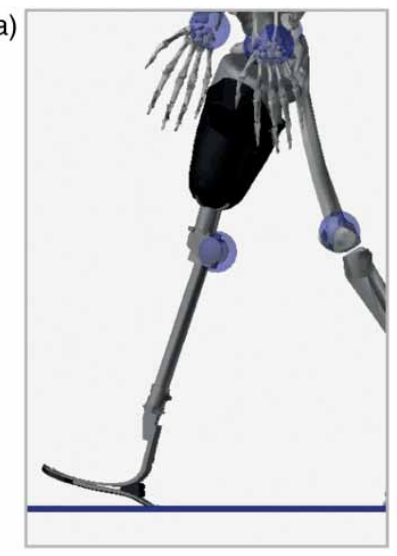

(b)

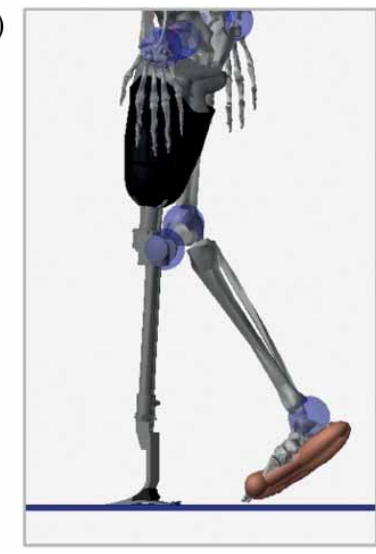

(c)

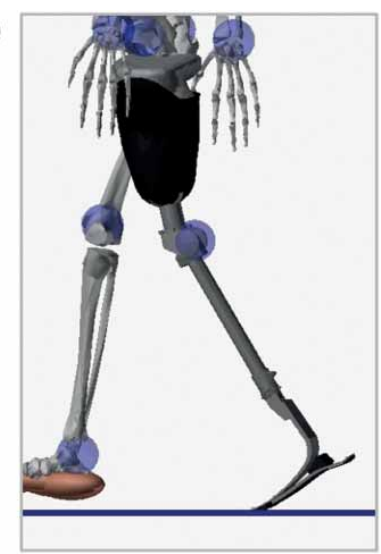

Fig. 4: Gait analysis model during stance phase: (a) initial loading response, (b) midstance, and (c) terminal stance. 


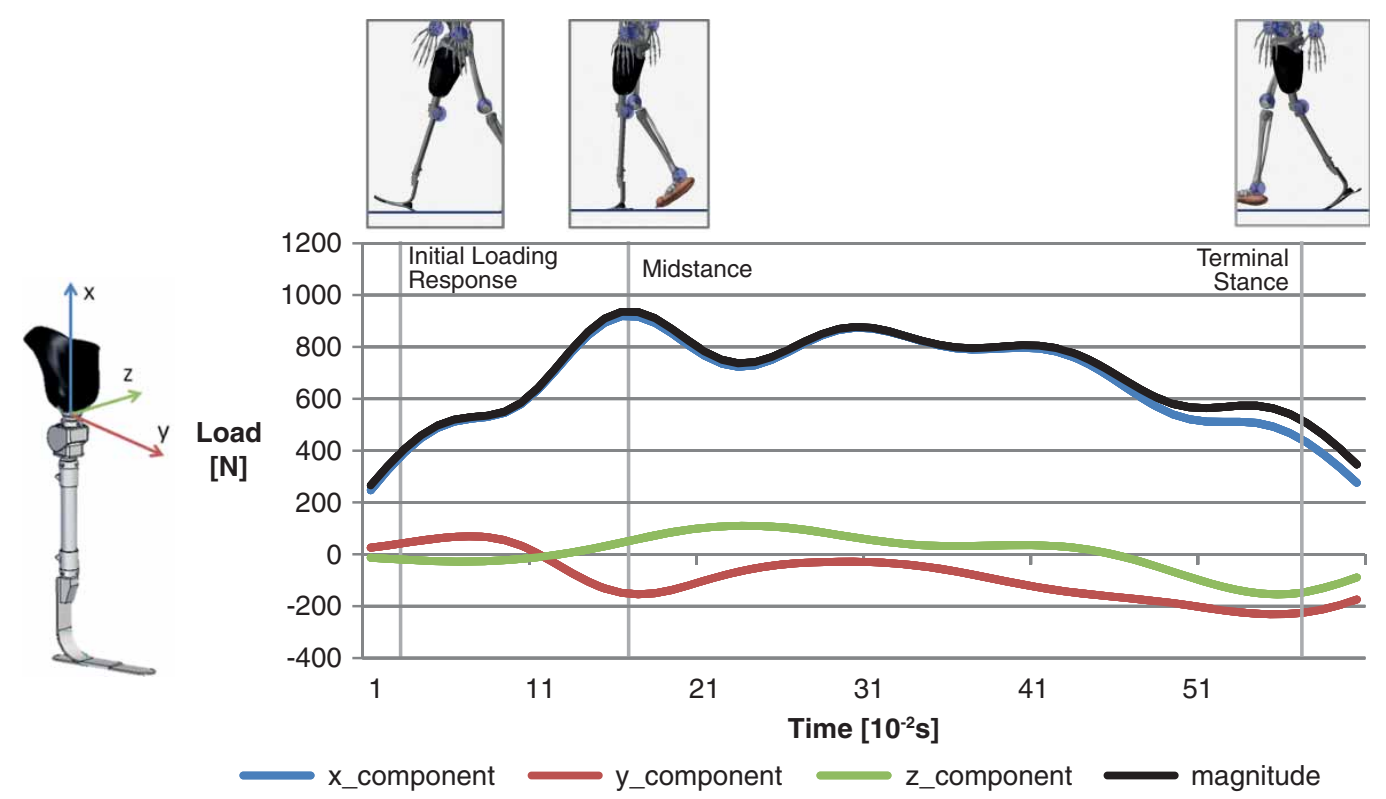

Fig. 5: Load components acting on the socket during stance phase: initial loading response, midstance, and terminal stance.

performing a sensitive analysis, following the conformity requirements of tetrahedral and triangular elements of the involved parts and without dramatically increasing analysis time. In particular, the sensitive analysis, carried out considering a seed value range of the residual limb from $8 \mathrm{~mm}$ to $3 \mathrm{~mm}$, has not showed results convergence; in fact, a finer mesh emphasizes a non-uniform contact between socket and the residual limb in numerical simulation.

\begin{tabular}{lccc}
\hline Transfemoral & Residual limb & Socket & Whole model \\
\hline Seed value $[\mathrm{mm}]$ & 6.3 & 8 & - \\
Nodes & 12176 & 920 & 13096 \\
Elements & 62279 & 1763 & 64042 \\
\hline
\end{tabular}

Tab. 1: Mesh characteristics: seed value, node number and elements number.

Regarding material properties, we first considered a linear, homogeneous and isotropic model to limit the computational time; then, we experimented a non-linear elastic approximation to get a better approx-imation of soft tissue behavior. Tab. 2 summarizes mechanical properties commonly found in literature for linear behavior characterization. According to some researches [13,19,21], we considered the socket and the bony structures as rigid bodies without los-ing crucial information about the pressure interface. The deformations of the socket and the bones can be neglected because Young's modulus of these parts is five orders of magnitude greater than soft tissue ones [13].

\begin{tabular}{|c|c|c|c|}
\hline $\begin{array}{l}\text { Part } \\
\text { Part }\end{array}$ & $\begin{array}{c}\text { Density } \\
{\left[\mathrm{Kg} / \mathrm{dm}^{3}\right]}\end{array}$ & $\begin{array}{c}\text { Young's } \\
\text { modulus [MPa] }\end{array}$ & $\begin{array}{l}\text { Poisson's } \\
\text { ratio }\end{array}$ \\
\hline Socket & 7.8 & Rigid body & \\
\hline Bones & 2.0 & Rigid body & \\
\hline Soft tissue & 1.48 & 0.2 & 0.49 \\
\hline
\end{tabular}

Tab. 2: Mechanical properties for linear behavior characterization (according to Jia et al. [10,11] and Lee at al. [14]).

Before simulating the stance phase using the loads achieved from gait analysis, we performed two phases corresponding to the deformation stages of soft tissues. The first step replicates the donning of residual limb into the socket and imposes a pre-stress on the residual limb, [24,26]. Then, the adjustment step follows to reach a better repositioning of the socket around the residual limb and to obtain maximum comfort. In the third and final step, the forces, computed by gait analysis, are applied as load to the centre of mass of the socket to simulate the single stance over the phase from Initial Loading Response to Terminal Stance.

Boundary conditions and loads have been defined according to the simulation step. The donning simulation is carried out fixing the upper residual limb surface and moving the socket proportionally to the residual limb length, causing the pre-stress on the external tissues. We chose to move the socket because relative adaptive movements are not known a priori and it limits computational costs. In the adjustment step, the upper residual limb surface in still fixed; the socket is free to translate and rotate in all directions 

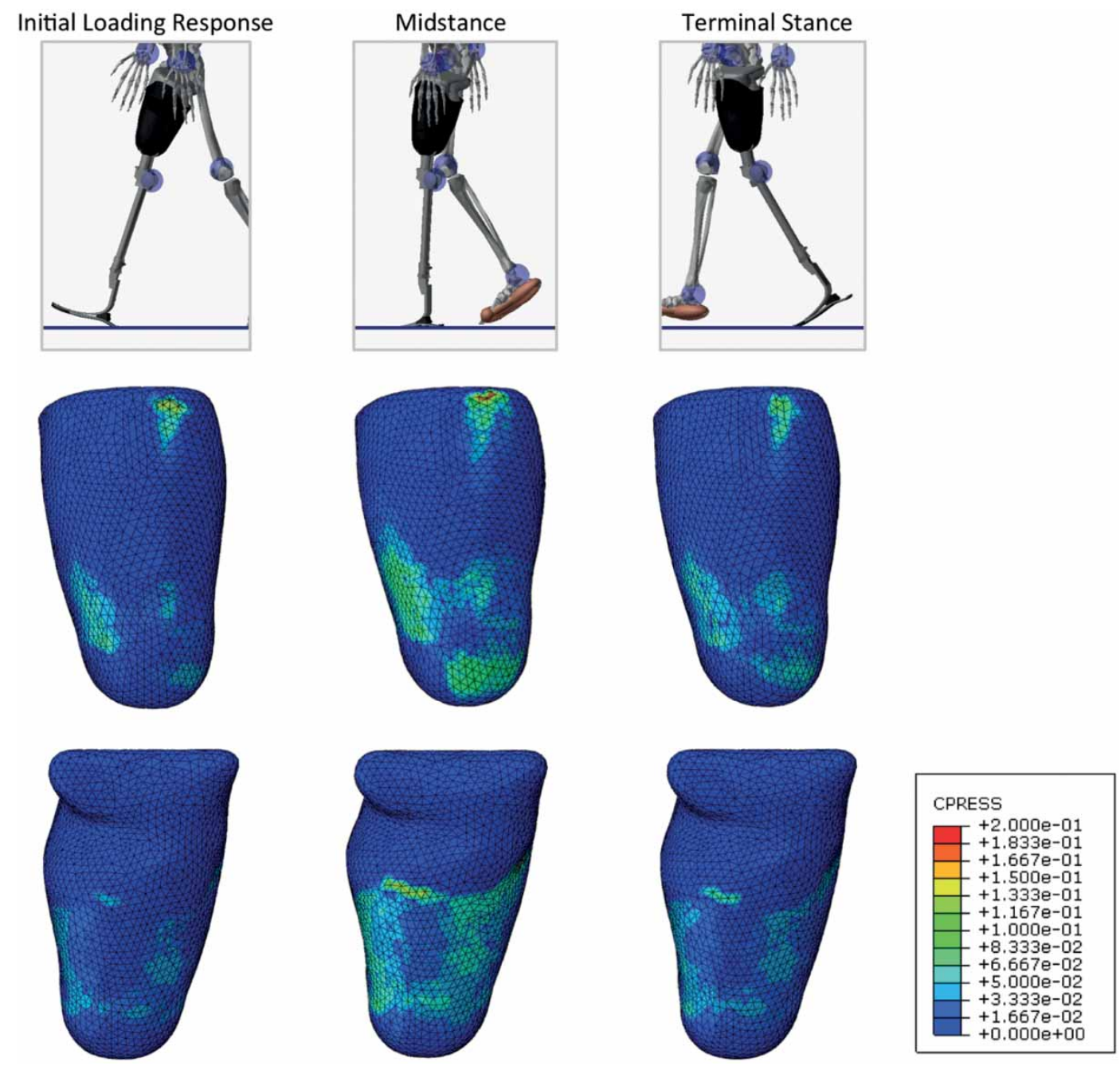

Fig. 6: Pressure distribution on residual limb surface during loading step in three different stance phases.

with the exception of the vertical one, which is kept locked until the load phase to prevent elastic spring back due to fitting. During these steps, no external load is applied. In the final step different load conditions have been studied. The dynamic load fluctuates over time according to the values achieved from the simulation of the patient ambulation. Socket translation is not applied instantly, but gradually during the analysis step to avoid excessive acceleration and then high mass inertia. The geometry of FE model, consequently the directions of forces and moments, determines considerably the analysis results: change in the residual limb posture affects the accuracy of interface forecast [27].

To model the interaction between the residual limb and socket, we adopted the automated surface-tosurface contact element since it is better than the traditional point-to point contact pairs, as reported by $\mathrm{Wu}$ et al. [22]. According to the master-slave contact formulation and hard contact relationship used in Abaqus, donning and adjustment steps are frictionfree, while during loading the friction coefficient is equal to 0.4 , within the range of value documented by Zhang and Mak [23].

\section{PRELIMINARY EXPERIMENTATION}

Main goal has been to verify the feasibility of the proposed approach. The automatic procedure has been tested considering as case study a unilateral transfemoral male amputee, 49 years old, $175 \mathrm{~cm}$ tall and $80 \mathrm{Kg}$ weight. The workstation used for the analysis of FE models has the following main features:

- CPU: Intel Xeon W3505@2.53 GHz;

- RAM: 12.0GB DDR3 @ 1333 Mh;

- Video card: Invidia Quadro FX 580;

- Operating System: Windows 7 Ultimate 64-bit.

The residual limb (soft tissues and bones) have been acquired using Magnetic Resonance Imaging, since it is the less invasive technique for the patient and permits to achieve a geometric model with reasonable 
accuracy. Then, the digital model of soft tissues and bones have been imported and aligned within the Socket Modeling Assistant - SMA, where the socket is modeled around the 3D model of the residual limb. At the same time, the prosthesis components are auto-matically chosen by the system from standard parts database according to patient's characteristics and then assembled. Following the procedure described in §3, we implemented the virtual patient wearing the prosthesis and forces derived from the simula-tion were exported in a text file. Then, we ran the script that retrieved necessary data both from SMA and above mentioned text file and automatically exe-cuted the FE analysis to get the pressure values over the residual limb surface.

Fig. 6 shows a comparison of pressure distribution during loading step in three different stance phases (Initial Loading Response, Mid-stance, and Ter-minal Stance) and considering linear behavior of soft tissues. Pressure values are associated to a color scale from blue to red, covering a range of fixed values from 0 to $200 \mathrm{kPa}$; the areas that exceed the maximum are colored in gray. The pressure distribution is well distributed and homogeneous, with the exception of external trochanter area, which seems to be overstressed. During the loading step the pressure distribution increases, as it should be, without exceeding $100 \mathrm{kPa}$ in most areas of the residual limb. Similar data can be found in literature, as described by Hong in [9]. However, even if preliminary results have been considered interesting by the prosthetist, we need to perform further activities to fully validate the approach and the FE models.

\section{CONCLUSIONS}

In this paper, we proposed an embedded simulation approach to analyze the interaction between socket and residual limb demonstrating its feasibility and focusing the attention on the transfemoral case that is the most challenging and less investigated compared with the transtibial one. The new design platform is centred on the digital model of the amputee and integrates the SMA prosthetic CAD system with FEA and the human modelling tools. By integrating the FE model with the patient's avatar and performing gait simulation, it is possible to determine the pressures distribution on the residual limb at any time of the stance phase during amputee's walking.

Preliminary results of the experimentation, obtained by the approach and the use of the amputee's avatar wearing the prosthesis, have been considered encouraging and promising to evaluate the socket-residual limb interaction. In addition, we verified that simulation results could offer precious information to characterize the pressure distribu-tion under different walking conditions and improve quality and comfort of the prosthetic socket.
Further developments and enhancements are necessary to make them easily usable and to validate the FE models. With regard to the last issue, in literature two main approcahes can be found. The former, the most diffuse, consists in comparing the computed pressure values with those measured with pressure transducers $[8,19,24]$, the latter in assess-ing the virtual soft tissue deformation with the real ones acquired by MRI during fitting or loading phase [12]. We have planned to adopt the first approach. In addition, we are considering non-linear characterization of soft tissues, specifically a Mooney-Rivlin hyperplastic model [28-29]. Non-linear characterization increases the complexity of the FE model and requires additional computational time, but it should permit to improve material behavior and final results. Therefore, we have planned to set an experimen-tal campaign to characterize material properties with indentation tests and to acquire real pressure values in the critical areas of the considered human district.

To conclude, the prosthetic CAD system associated with finite elements analysis and virtual human applications should permit to reduce number of phys-ical prototypes and lower the psychological impact on the life of the patient; in fact, the virtual approach allows carrying out a several tests faster than the traditional socket development process. Furthermore, even if we put the attention on lower limb pros-thesis, we feel confident about the extension of the approach to other kind of prostheses, primarily for those types in which a patient specific modelling is required, starting from 3D anatomical models.

\section{ACKNOWLEDGEMENTS}

The authors want to thank Fondazione Cariplo (Intesa Bank, Italy), which co-funded this research project.

\section{REFERENCES}

[1] AutoSculpt, http://www.infinitycadsystems. com, Infinity CAD Systems.

[2] Baars, E. C. T.; Geertzen, J. H. B.: Literature review of the possible advantages of silicon liner socket use in trans-tibial prostheses, Prosthetics and Orthotics International, 29(1), 2005, 27-37.

[3] Canfit, http://www.vorum.com, Vorum Research Corporation.

[4] Colombo, G.; Facoetti, G.; Rizzi, C.: A digital patient for computer-aided prosthesis design, Interface Focus, 3(2), 2013.

[5] Colombo, G.; Facoetti, G.; Morotti, R.; Rizzi, C.: Physically based modelling and simulation to innovate socket design, Computer-Aided Design and Applications, 8(4), 2011, 617-631.

[6] Duffy, V. D.: Digital Human Modeling, Proc. First International Conference on Digital Human Modeling, ICDHM 2007, Held as Part of HCI International 2007, Springer, 2007. 
[7] Facoetti, G.; Gabbiadini, S.; Colombo, G.; Rizzi, C.: Knowledge-based system for guided modeling of sockets for lower limb prostheses, Computer-Aided Design and Applications, 7(5), 2010, 723-737.

[8] Goh, J. C. H.; Lee, R. S.; Toh, S. L.; Ooi, C. K.: Development of an integrated CAD-FEA process for below-knee prosthetic sockets, Clinical Biomechanics, 20(6), 2005, 623-629.

[9] Hong, J. H.: Effect of hip moment on socket interface pressure during stance phase gait of transfemoral amputee, Gait \& Posture, 24, Supplement 2(0), 2006, S259-S261.

[10] Jia, X. H.; Zhang, M.; Lee, W. C. C.: Load transfer mechanics between trans-tibial prosthetic socket and residual limb - dynamic effects, Journal of Biomechanics, 37(9), 2004, 13711377.

[11] Jia, X. H.; Zhang, M.; Li, X. B.; Lee, W. C. C.: A quasi-dynamic nonlinear finite element model to investigate prosthetic interface stresses during walking for trans-tibial amputees, Clinical Biomechanics, 20(6), 2005, 630-635.

[12] Kovacs, L.; Eder, M.; Volf, S.; Raith, S.; Pecher, M.; Pathak, H.; Müller, C.; Gottinger, F.: PatientSpecific Optimization of Prosthetic Socket Construction and Fabrication Using Innovative Manufacturing Processes: A Project in Progress, Proc. Materialise World Conference 2010.

[13] Lee, W. C. C.; Zhang, M.: Using computational simulation to aid in the prediction of socket fit: A preliminary study, Medical Engineering \& Physics, 29(8), 2007, 923-929.

[14] Lee, W. C. C.; Zhang, M.; Jia, X. H.; Cheung, J. T. M.: Finite element modeling of the contact interface between trans-tibial residual limb and prosthetic socket, Medical Engineering \& Physics, 26(8), 2004, 655-662.

[15] Magnenat-Thalmann, N.; Thalmann, D.: Handbook of virtual humans, John Wiley \& Sons, Chichester, UK., 2004.

[16] Mak, A. F. T.; Zhang, M.; Boone, D. A.: Stateof-the-art research in lower-limb prosthetic biomechanics-socket interface: A review, Journal of Rehabilitation Research and Development, 38(2), 2001, 161-173.

[17] OMEGA, http://www.willowwoodco.com, The Ohio Willow Wood Company.

[18] R4D-CADCAM, http://www.rodin4d.com, Rodin4D - Lagarrigue Group.
[19] Sanders, J. E.; Daly, C. H.: Normal and shear stresses on a residual limb in a prosthetic socket during ambulation - comparison of finite-element results with experimental measurements, Journal of Rehabilitation Research and Development, 30(2), 1993, 191-204.

[20] Sewell, P.; Noroozi, S.; Vinney, J.; Andrews, S.: Developments in the trans-tibial prosthetic socket fitting process: a review of past and present research, Prosthetics and Orthotics International, 24(2), 2000, 97-107.

[21] SilverThorn, M. B.; Childress, D. S.: Parametric analysis using the finite element method to investigate prosthetic interface stresses for persons with trans-tibial amputation, Journal of Rehabilitation Research and Development, 33(3), 1996, 227-238.

[22] Wu, C. L.; Chang, C. H.; Hsu, A. T.; Lin, C. C.; Chen, S. I.; Chang, G. L.: A proposal for the pre-evaluation protocol of below-knee socket design - Integration pain tolerance with finite element analysis, Journal of the Chinese Institute of Engineers, 26(6), 2003, 853-860.

[23] Zhang, M.; Mak, A. F. T.: In vivo friction properties of human skin, Prosthetics and Orthotics International, 23(2), 1999, 135-141.

[24] Zhang, M.; Roberts, C.: Comparison of computational analysis with clinical measurement of stresses on below-knee residual limb in a prosthetic socket, Medical Engineering \& Physics, 22(9), 2000, 607-612.

[25] Zhang, M.; Mak, A. F. T.; Roberts, V. C.: Finite element modelling of a residual lower-limb in a prosthetic socket: a survey of the development in the first decade, Medical Engineering \& Physics, 20(5), 1998, 360-373.

[26] Zhang, M.; Lord, M.; Turnersmith, A. R.; Roberts, V. C.: Development of a nonlinear finite-element modeling of the below-knee prosthetic socket interface, Medical Engineering \& Physics, 17(8), 1995, 559-566.

[27] Zhang, M.; Turner-Smith, A. R.; Tanner, A.; Roberts, V. C.: Clinical investigation of the pressure and shear stress on the trans-tibial stump with a prosthesis, Medical Engineering \& Physics, 20(3), 1998, 188-198.

[28] Zheng, Y. P.; Mak, A. F. T.; Leung, A. K. L.: State-of-the-art methods for geometric and biomechanical assessments of residual limbs: A review, Journal of Rehabilitation Research and Development, 38(5), 2001, 487-504. 